\title{
A Case Study on the Selection of Materials for Eye Lenses
}

\author{
Fehim Findik \\ Department of Mechanical Engineering, Faculty of Engineering and Natural Sciences, International University of Sarajevo, \\ Hrasnicka Cesta 15, 71000 Sarajevo, Bosnia and Herzegovina
}

Correspondence should be addressed to Fehim Findik, ffindik@ius.edu.ba

Received 16 January 2011; Accepted 1 March 2011

Academic Editors: G. Exadaktylos and T. Y. Ng

Copyright (c) 2011 Fehim Findik. This is an open access article distributed under the Creative Commons Attribution License, which permits unrestricted use, distribution, and reproduction in any medium, provided the original work is properly cited.

A case study on the selection of materials for eye lenses is reported by means of Ashby's materials selection diagrams. In this study, 6 polymer materials of PMMA, PC, PS, PVC, PET, and PA are reviewed and their properties are found from the selection diagrams of Ashby and compared in a table. The final results confirmed that PMMA (acrylic) is the best material for eye lenses. The outcome also showed that the present material is environmental friendly being nontoxic and recyclable.

\section{Introduction}

A contact lens is a lens worn in front of the eye, mainly used to treat myopia, hyperopia, astigmatism, and presbyopia. Glasses or "spectacles" are worn on the face a short distance in front of the eye. Contact lenses are worn directly on the surface of the eye. Intraocular lenses are surgically implanted most commonly after cataract removal, but recently for purely refractive purposes. Myopia (near sightedness) requires a divergent lens, whereas hyperopia (far sightedness) requires convergent lens.

Contact lenses usually serve the same corrective purpose as conventional glasses but are lightweight and virtually invisible-many commercial lenses are tinted a faint blue to make them more visible when immersed in cleaning and storage solutions. Some cosmetic lenses are deliberately colored to alter the appearance of the eye.

It has been estimated that 125 million people use contact lenses worldwide (2\%) [1], including 28 to 38 million in the United States [1, 2] and 13 million in Japan [3]. The types of lenses used and prescribed vary markedly between countries, with rigid lenses accounting for over $20 \%$ of currently prescribed lenses in Japan, The Netherlands, and Germany but less than 5\% in Scandinavia [1].

People choose to wear contact lenses for many reasons, often due to their appearance and practicality [4]. When compared to spectacles, contact lenses are less affected by wet weather, do not steam up, and provide a wider field of vision. They are more suitable for a number of sporting activities. Additionally, ophthalmological conditions such as keratoconus and aniseikonia may not be accurately corrected with glasses.

Many types of contact lenses are available. The type of contacts you use depends on your particular situation. Your eye doctor will be able to help you choose from the following types of lenses.

Rigid or "hard" contacts were the first lenses; they were developed in the 1960s. They are made of a type of plastic called PMMA (polymethyl methacrylate), which is very durable but does not allow oxygen in the air to directly reach the cornea. When the eye blinks, the lens moves, which allows the oxygen dissolved in the tears to reach the cornea. Rigid lenses are the least comfortable type of contacts and are not really used anymore. However, some people still prefer them for their durability and lower cost.

Gas-permeable lenses are also known as "RGPs." They are newer rigid or "hard" lenses made of plastics combined with other materials, such as silicone and fluoropolymers, which allow oxygen in the air to pass directly through the lens. For this reason, they are called "gas permeable."

Soft contact lenses are made of plastic materials that incorporate water. The water makes them soft and flexible, as well as allowing oxygen to reach the cornea. More than $75 \%$ of contact lens wearers in the United States use soft lenses.

Generally, contacts should be removed at bedtime due to risk of infection and risk of contact lens intolerance. In this 
paper, a case study on the selection of materials for eye lenses is given using Ashby's selection diagrams.

\section{Materials for Eye Lenses}

Up until the late 1970s, contact lenses were made from two materials. Hard contact lenses were made of polymethyl methacrylate (PMMA), while the soft contact lenses were made of a hydrated polymer, hydroxyethylmethacrylate (HEMA), which contained $37.8 \%$ water by weight. These lenses provided clear vision and comfort, but there was a problem. These lenses did not allow oxygen to reach the cornea. Because of this, the cornea could change, adversely, in some contact lens wearers.

PMMA is now obsolete and is replaced with rigid plastics, mostly hydrophobic materials with high oxygen permeability. These lenses are called rigid gas permeable (RGP) lenses. For the manufacture of soft lenses, HEMA is being replaced by polymers which contain as much as $80 \%$ water. These soft lenses, often called hydrogels because of the amount of water, and they have high oxygen transfer while retaining shape despite high water content. These new materials used in the manufacture of contact lenses as well as thinner lenses and greater oxygen transfer have reduced corneal issues, but there are still other possible complications.

The new materials have also been instrumental in the creation of disposable contact lenses in a variety of types. Disposable contact lenses come in monthly, bi-monthly, weekly, and even daily disposable types. The thinner materials make wearing these contact lenses more comfortable, and the cost of contact lenses has been reduced. This allows for close to maintenance-free contact lens wear.

\section{The Selection}

Material selection is the process of choosing the best material for a particular design. In mechanical design, material selection enters at every stage of the design process [5] and is interrelated and as important as design and manufacture. In the total design model [6], material selection is rated as one of the fundamental parameters along with market investigation, product design specification, component design, design analysis, manufacture, and assembly. Other models of product design $[7,8]$ also emphasize the importance of correct material selection. Ashby [8] has shown that the material requirement changes as design moves from concept through embodiment to detail. The computer-based system developed from these ideas is known as the Cambridge Engineering System (CES), originally Cambridge Material Selector (CMS). The system uses material selection charts, which are a way of displaying material property data through the use of optimized procedures. The selection works by evaluating performance indices, derived from mechanical analysis, which, if maximized, optimize performance.

In this study, in order to find the better material for cataract problem, eye lens materials are evaluated and selected for this problem. The following steps are used for the materials selection.

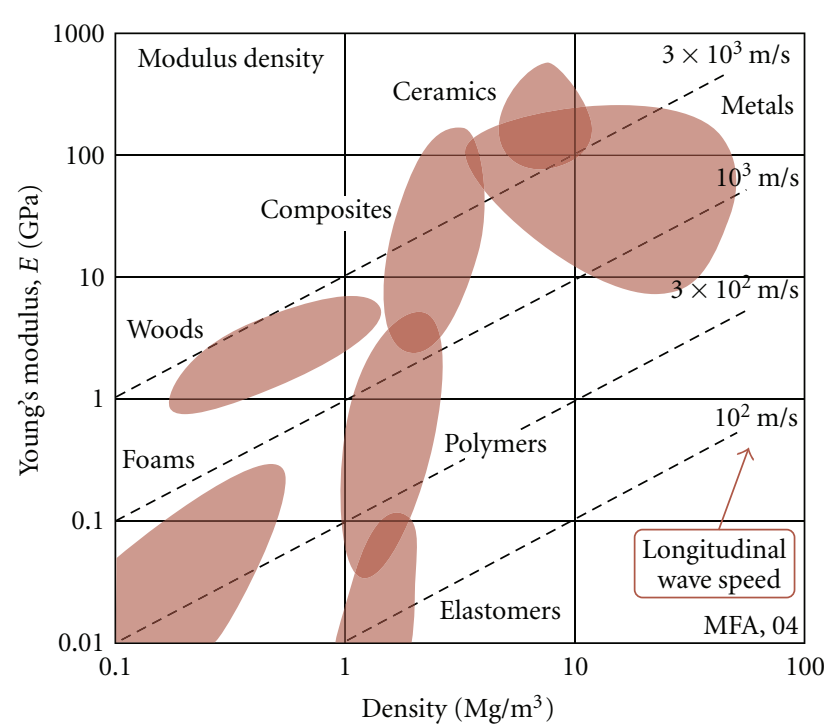

FIGURE 1: The idea of a materials property chart: Young's modulus, $E$, is plotted against the density, $\rho$, on log scales [8].

3.1. Analysis of the Required Properties for Eye Lenses. It is known that eye lenses should have the following properties:

(i) easy manufacturing,

(ii) must be transparent,

(iii) light (low density),

(iv) cheap (reasonable price),

(v) bio compatible,

(vi) medium fracture toughness,

(vii) lower elastic modulus.

3.2. Range of Alternative Materials. Considering the abovementioned properties for eye lenses, it is possible to give the candidacy for the following materials:

(i) polymethyl methacrylate (PMMA: Acrylic),

(ii) polycarbonate (PC),

(iii) polystyrene (PS),

(iv) polyvinylchloride (PVC),

(v) polyesters (PET, PBT),

(vi) polyamide (PA: Naylon).

3.3. Evaluation of the Candidates. In order to evaluate the candidate materials, we can make a table showing the candidate materials in the first line and the properties are in the first column. After that, we can read their numerical values from Ashby's selection diagrams [8]. The final results are shown in Table 1 using Ashby's materials properties (Figures 1 and 2). In Table 1, the numerical values are shown in a range and in the parenthesis its score is shown between 1 to 10 points. If the material is fully consistent with the required properties, 10 points are given as a score, otherwise in a worse condition only 1 point can be given for the candidate material. 
TABLE 1: Candidate materials for eye lenses and their scores.

\begin{tabular}{|c|c|c|c|c|c|c|}
\hline \multirow{2}{*}{ Property } & \multicolumn{6}{|c|}{ Material } \\
\hline & PMMA & $\mathrm{PC}$ & PS & PVC & PET & PA \\
\hline $\begin{array}{l}\text { Fracture toughness } \\
\left(\mathrm{MPa} \cdot \mathrm{m}^{1 / 2}\right)\end{array}$ & $0.70-1.6(5 p)$ & $2.1-4.60(8 p)$ & $0.70-1.1(3 \mathrm{p})$ & $1.46-5.12(9 p)$ & $1.01-1.70(5 p)$ & $0.58-8.03(5 \mathrm{p})$ \\
\hline Density $\left(\mathrm{Mg} / \mathrm{m}^{3}\right)$ & $1.16-1.22(5 \mathrm{p})$ & $1.14-1.21(5 \mathrm{p})$ & $1.04-1.05(7 \mathrm{p})$ & $1.30-1.58(3 \mathrm{p})$ & $0.10-1.40(10 p)$ & $1.00-1.42(5 \mathrm{p})$ \\
\hline Easy manufacturing & $+++(10 p)$ & $++(6 \mathrm{p})$ & $+++(10 p)$ & $++(6 \mathrm{p})$ & $+++(10 p)$ & $+++(10 p)$ \\
\hline Price $(\$ / k g)[9]$ & $1.70-2.40(8 p)$ & $3.80-4.30(5 \mathrm{p})$ & $1.30-1.60(9 \mathrm{p})$ & $1.00-1.20(10 \mathrm{p})$ & $1.44-2.51(8 p)$ & $2.90-11.50(1 \mathrm{p})$ \\
\hline Transparency [9] & Transparent $(10 \mathrm{p})$ & Transparent $(10 p)$ & Opaque (1 p) & $\begin{array}{l}\text { Transparent to } \\
\text { opaque ( } 5 \mathrm{p})\end{array}$ & Opaque (1 p) & Opaque (1 p) \\
\hline Elastic modulus (GPa) & $2.24-3.80(9 p)$ & $2.21-2.44(10 p)$ & $2.28-3.34(9 \mathrm{p})$ & $2.14-4.14(9 \mathrm{p})$ & $0.30-4.41(10 \mathrm{p})$ & $0.67-4.51(10 \mathrm{p})$ \\
\hline Total score & $47 \mathrm{p}$ & $44 \mathrm{p}$ & $39 p$ & $42 \mathrm{p}$ & $44 \mathrm{p}$ & $32 \mathrm{p}$ \\
\hline
\end{tabular}

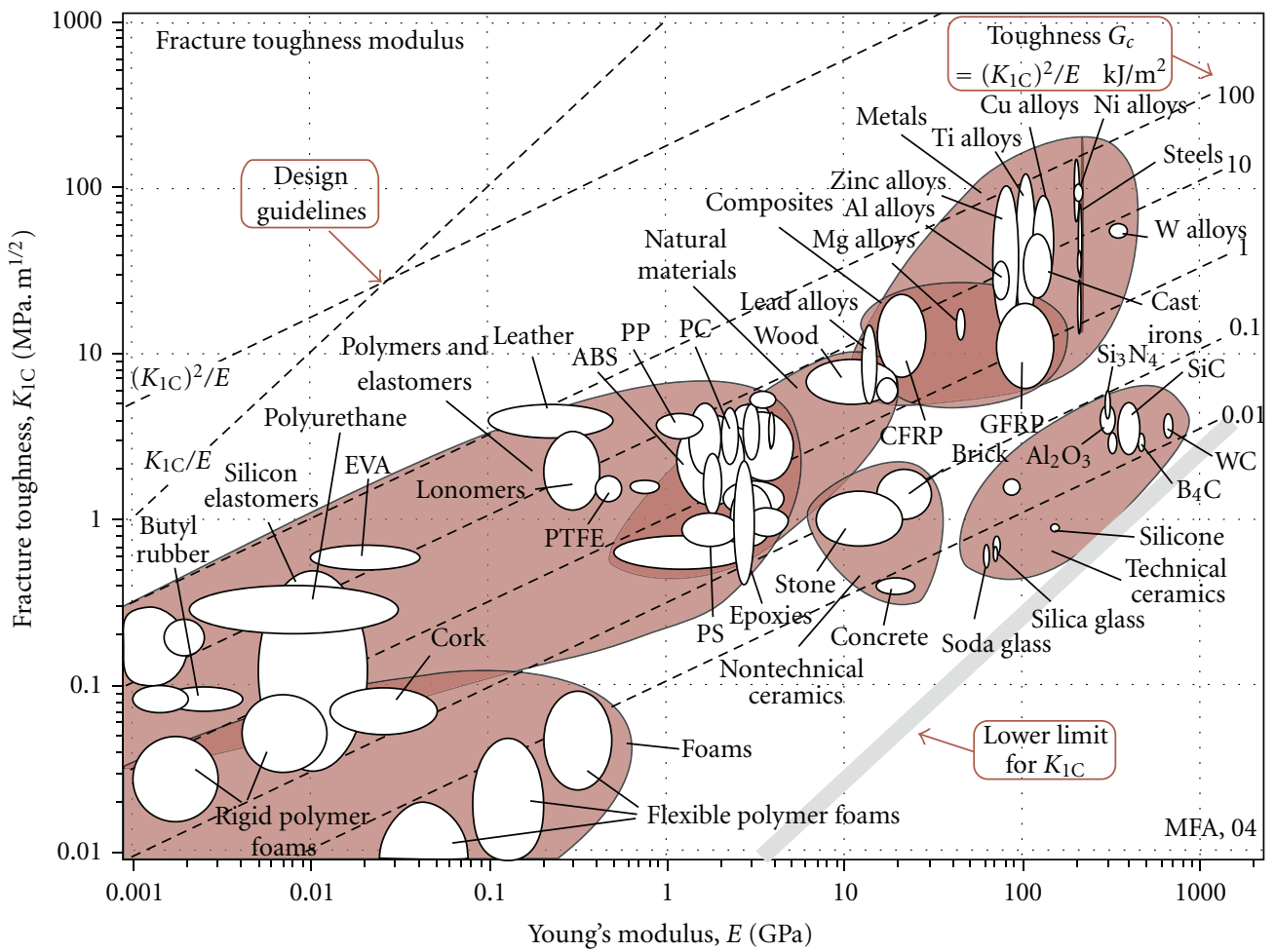

Figure 2: Fracture toughness, $K_{\mathrm{IC}}$, plotted against Young's modulus, $E$ [8].

3.4. Selection of the Best Material for Eye Lenses. It is seen from Table 1 that the score of PMMA is 47 points and therefore its performance is maximum. PMMA (acrylic) has the best performance due to its easy manufacturing, transparency, suitable elastic modulus, and also its reasonable price. In the second order, PC and PET are seen within the same score of 44 . However, PET must be cancelled due to its opacity. PC is a little cheaper than Acrylic, but it has some manufacturing difficulties [9]. Therefore, it picks up a lower point than Acrylic. In addition, all materials should be biocompatible in the body and all polymeric materials used in eyes have biocompatibility. Therefore, no problem is faced about this issue.

\section{Postscript}

Acrylic, or PMMA, is the thermoplastic that most closely resembles glass in transparency and resistance to weathering. It is hard and stiff as polymers go, easy to polish but sensitive to stress concentrations. It shares with glass certain fragility, something that can be overcome by blending with acrylic rubber to give a high-impact alloy (HIPMMA). PVC can be blended with PMMA to give tough, durable sheets. Acrylic is available as a sheet, rod, or tube and can be shaped by casting or extrusion [9]. Also, recycle potential and resistance to weathering of acrylic are high; therefore eye lenses are largely produced from acrylic or PMMA. 


\section{Conclusion}

In this paper, a case study on the selection of materials for eye lenses is given using Ashby's selection diagrams. In this work, 6 polymer materials of PMMA, PC, PS, PVC, PET, and $\mathrm{PA}$ are taken into consideration and their properties are found from the selection diagrams of Ashby and compared in Table 1. The final results showed that PMMA (acrylic) is the best for eye lenses. The outcome also supported that PMMA material is good for environmental considerations (nontoxic and recyclable).

\section{References}

[1] J. Barr, “2004 Annual Report,” Contact Lens Spectrum, January 2005.

[2] D. Farley, Keeping an Eye on Contact Lenses: Safety, Options Shape Contact Lens Decisions, U.S. Food and Drug Administration: FDA Consumer, 1998;.

[3] National Consumer Affairs Center of Japan. NCAC News, NCAC News, vol. 12, no. 4, 2001

[4] J. L. Sokol, M. G. Mier, S. Bloom, and P. A. Asbell, "A study of patient compliance in a contact lens-wearing population," CLAO Journal, vol. 16, no. 3, pp. 209-213, 1990.

[5] J. A. Charles, F. A. A. Crane, and J. A. E. Furness, Selection and Use of Engineering Materials, Butterworth-Heinemann, Oxford, UK, 3rd edition, 1997.

[6] S. Pugh, Total Design: Integrated Methods for Successful Product Engineering, Addison Wesley, Wokingham, UK, 1991.

[7] G. Pahl and W. Beitz, Engineering Design: A Systematic Approach, The Design Council, London, UK, 1984, Edited by K. Wallace.

[8] M. F. Ashby, Materials Selection in Mechanical Design, Butterworth-Heinemann, Oxford, UK, 3rd edition, 2005.

[9] M. F. Ashby and K. Johnson, Materials and Design, Elsevier, London, UK, 3rd edition, 2005. 

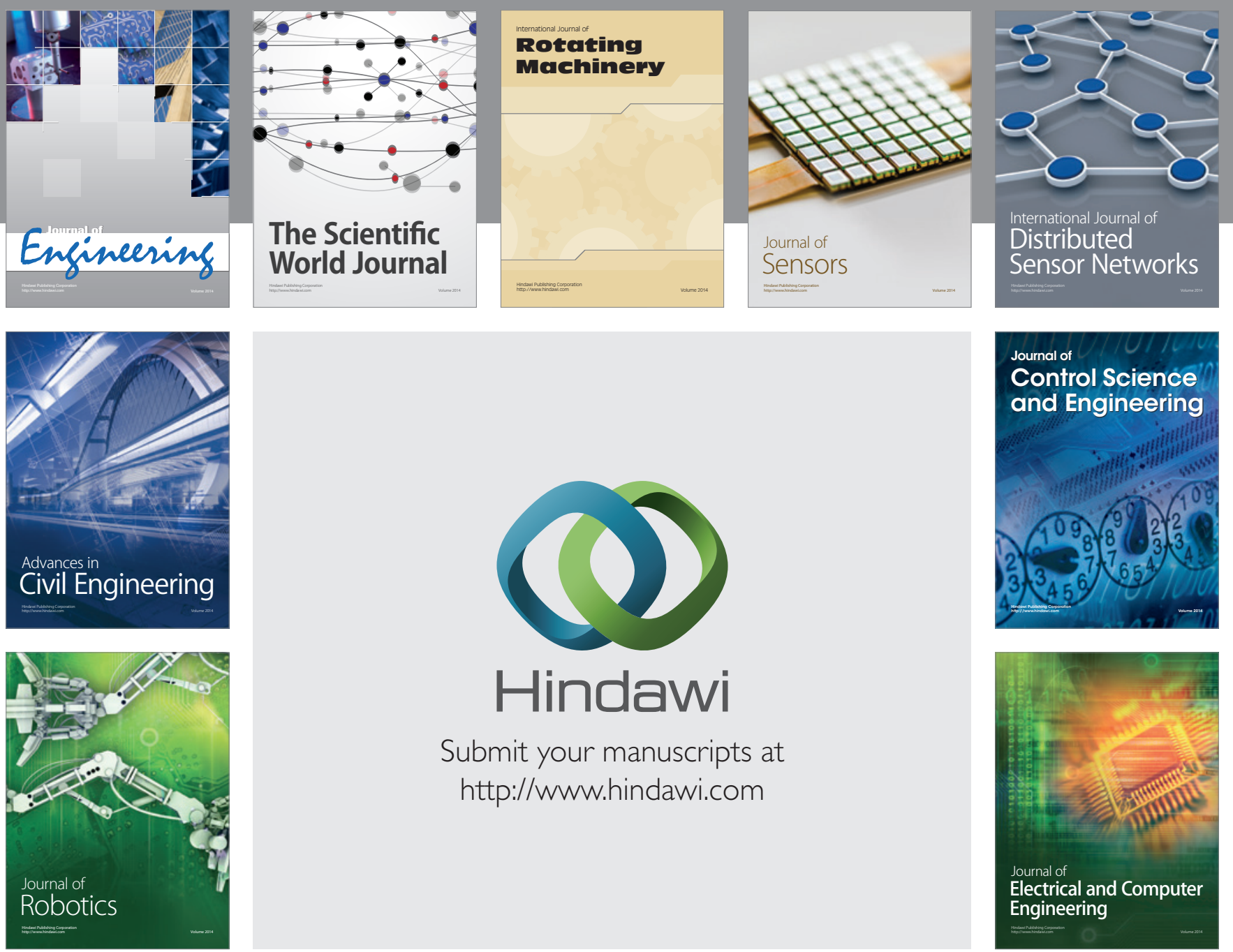

Submit your manuscripts at

http://www.hindawi.com
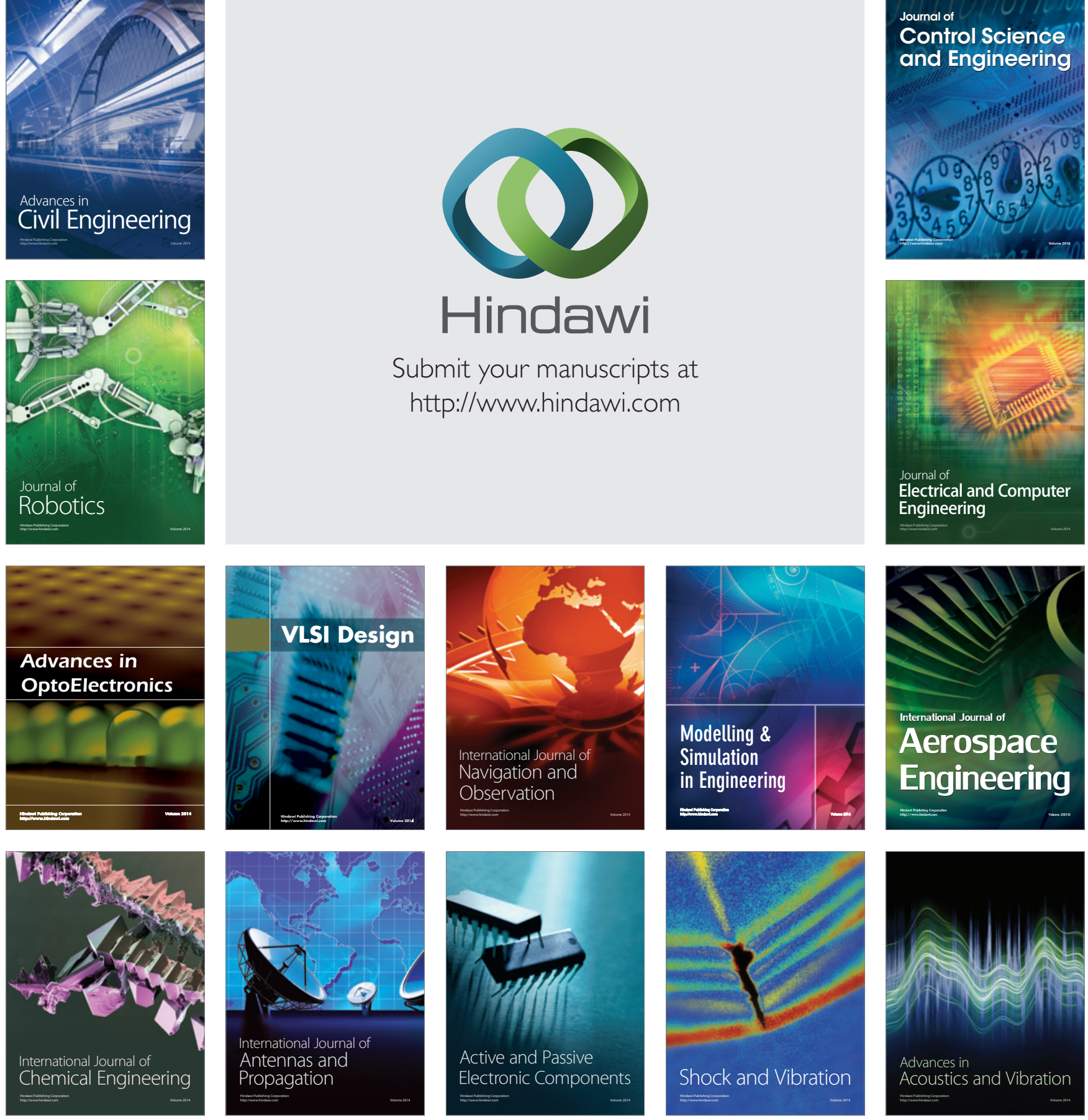